\title{
In defence of the depot clinic
}

\author{
The consumers' opinion
}

DAVId ANDerson, Senior Registrar in Psychiatry, Department of Psychogeriatrics, Royal Liverpool Hospital, Prescot Street, Liverpool L7 8XP (correspondence); ANGela LeadBetTer, Community Psychiatric Nurse, Sefton General Hospital; and Bernie Williams, Community Psychiatric Nurse, Royal Liverpool Hospital

The depot clinic has long been part of the traditional British psychiatric service. With moves towards care in the community many of the traditional, particularly hospital based, methods of service delivery are becoming unfashionable. The depot clinic has been criticised (Beveridge, 1987) for being out of date, not geared to the needs of the patient, inaccessible and unable to provide personalised care, especially regarding the family factors recognised to be important in the management of chronic psychosis, and is portrayed as a facility designed for the convenience of the staff not the patient. This has led to suggestions for alternative methods of administering treatment to these patients, e.g. at home, from GP, health centre based.

Considering the consumers' view has been identified as an essential component of good community care (Murphy, 1988) yet their views are rarely sought when planning service provision, and dissatisfaction with existing services often reflects the assumptions and opinions of the planners. Although we share many of the reservations of the depot clinic, our experience suggested that a significant proportion of patients felt positively about them and might even prefer the clinic to some of the alternatives proposed. We therefore sought the consumers' view and feel that the results, many of which surprised us, are worth reporting.

\section{The study}

A simple 16 item questionnaire was given to all patients attending two depot clinics serving the same health district over the same one month period. The questions were direct, aimed at eliciting general attitudes and requiring a single yes or no response, e.g. "Do you enjoy attending the clinic?"; "Are you given enough time to see the doctor about any problem?". Patients completed a questionnaire in private and their responses remained anonymous. Patients returning the questionnaire were recorded to ensure that no individual was included more than once.

\section{The clinics}

Clinic $A$ - the depot clinic of the major teaching hospital serving a deprived inner city catchment area of approximately 70,000 population. It is centrally placed and easily accessible from all points of the area it serves. The community nurses organise and run the clinic, which is held in the unit's day hospital. The day hospital vacates its dining area for the afternoon of the clinic to be used as a waiting area for depot patients. The clinic operates from 1-5 p.m., with no appointment times, and is staffed by an average of two CPNs (there were three serving the area at the time of the study). There are only two rooms for administering injections and talking privately with patients. All areas are cramped and far from satisfactory. There are no facilities for light refreshment, which has to be obtained by patients visiting the WRVS canteen in another area of the hospital. Medical input is provided by the day hospital registrar, usually a senior trainee, although patients are only seen at the request of the CPN. No patients are seen routinely, although some will also attend the out-patient clinic. Patient attendance is noted and non-attendance is followed by a visit from the CPN.

Clinic $B$-based in a district general hospital serving a catchment area of approximately 110,000 . The catchment area includes deprived areas of the city and surrounding districts. The hospital is peripherally situated and difficult to get to for a number of patients, necessitating a substantial journey, often by public transport. The clinic is held in its own building which also houses the CPNs' offices but is used for other purposes during the rest of the week. There is adequate space, although it is drably decorated, and there are a number of rooms available for patients to be seen in some privacy. The clinic operates from 1-4 p.m., no appointment times, and staffed by an average three CPNs (there were five covering the area at the time of the study). Whenever possible the patients' community nurse will administer treatment, although this is far from guaranteed. The clinic has intermittently had volunteer helpers 
(but not at the time of the study) and has facilities to provide tea free of charge. A GP clinical assistant routinely sees a small list of patients weekly, selected by the CPNs, aiming to review all patients over a period of time and visiting some patients at home if they fail to attend or the CPN is concerned. All patients register, the staff endeavouring to see them in order of arrival. If a patient fails to attend they will be visited within $24-48$ hours. In recent months there have been attempts to generate a social milieu with the development of a social club organising events for patients, although this has yet to become established. A total of 168 patients ( 77 patients from clinic $A$ and 91 from clinic B) completed the questionnaire.

Of the 168 patients, $80 \%$ were unmarried though only $38 \%$ lived alone. Twenty-seven per cent attended a day centre, $63 \%$ reported meeting friends weekly (outside of the clinic) and 69\% saw family members at least fortnightly.

Comparing responses from the two clinic samples showed a striking similarity, with only two items distinguishing them statistically. These were "Is it difficult to get to the clinic?", producing a yes response from $10 \%$ of clinic A but $31 \%$ of clinic $B(P<0.01)$; and "Do you meet friends at the clinic?", producing a yes response from $71 \%$ of clinic A but $55 \%$ of clinic B $(P<0.05)$. These are, perhaps, not surprising given the peripheral location of clinic $B$ in its catchment area, which is also more spread out than that of clinic $\mathbf{A}$.

Overall, only $21 \%$ of patients found difficulty getting to the clinic while $32 \%$ said it was "inconvenient" to attend; $31 \%$ positively "disliked" attending while $60 \%$ positively "enjoyed" attending and $51 \%$ considered attending the clinic to be a "nice day out". Sixty-three per cent stated they met 'friends' at the clinic. Seventy-six per cent felt they were given sufficient time to see the doctor about any problems and $82 \%$ similarly thought they had enough time to see the nurse. Thirty-nine per cent of patients would have preferred to receive their injection at home while only $17 \%$ would have chosen to receive their treatment in their GP's surgery, even though $70 \%$ had seen their GP within the preceding three months. Twelve per cent opted for either home treatment or GP surgery before the clinic while $56 \%$ rejected both alternatives in favour of the clinic.

\section{The findings}

The two clinics demonstrate how varied depot clinics can be even within the same health district. They both clearly have a number of deficiencies and illustrate how little priority is given to these facilities, even though they provide a vital sontact with some of the most seriously ill and handicapped of all psychiatric patients. It is in this neglected context that depot clinics are being judged. It is then surprising to find that the majority of our patients enjoyed attending $(60 \%)$, preferred to receive their treatments in the clinic rather than at home $(61 \%)$, expressed an emphatic preference for the clinic as opposed to the GP's surgery $(83 \%)$. Most patients found no difficulty getting to the clinic (79\%) and found attendance convenient (68\%). Accepting the lack of adequate numbers of staff supervising the clinic and the frequently large numbers of patients attending, it was surprising to find that $76 \%$ felt they were given enough time to see the doctor and $82 \%$ the nurse.

The questionnaire offered patients the opportunity to make comments on improving the clinic and $40 \%$ did so. Sixty-three per cent of requests from clinic A were for light refreshments to be available. This was not an issue in clinic B where one sixth of requests were for the return of the volunteer helpers, one quarter for music in the social areas and the remainder various suggestions including improved furnishings and decor, more community nurses, reimbursement of bus fares (one patient, rather poignantly, suggested that a collection should be made each week for those who had to pay) and one enlightened patient suggested leaflets should be available which educate relatives about their illness. Cost of travelling to and from the clinic is an important issue for some patients who are unable to claim travelling expenses and can amount to a significant sum for those on low income. We believe patients attending clinic this frequently should automatically be eligible for refunds.

Alternative methods of administering treatment to these patients are growing in popularity. In this district it has, particularly, been suggested that delivery of care should be moved to local health centres although still administered by CPNs. There would seem little advantage to the patients if this change took place (ease of accessibility would be improved for a proportion of clinic B attenders) and our results suggest it would be unpopular with them. It would, it is suggested, facilitate transfer of the drug bill from hospital to the family practitioner budget and one suspects this may be an important motivation behind the proposal.

There are a number of disadvantages to moving the clinic from the hospital site which we believe to be important. There will be greater difficulty getting patients seen by medical staff, the GP will naturally become the accessible medical practitioner but there is an alarming lack of enthusiasm among family practitioners to become involved with this growing group of patients (Kean \& Fahy, 1982). We believe that distancing the clinic from the hospital geographically will distance it emotionally from the inpatient and out-patient unit and though many would see this as progress we suggest this link is extremely important. Many chronically mentally ill patients form invaluable emotional bonds with the unit that treats them and great effort is made to create rapport and establish these bonds. The more the service is 
seen as a single functioning unit serving different needs at different times, the more these emotional bonds will generalise to all aspects of care. There are, in addition, purely practical considerations such as ease of obtaining the depot prescription, cost effectiveness, efficient use of limited CPN time, record keeping, patient registers etc.

Where we believe the depot clinic is better, but which we have purposefully avoided discussing so as not to detract from issues of care, we feel to be of greater fundamental importance and often ignored. Depot clinics are frequently perceived as filling stations where patients fill up with $50 \mathrm{mg}$ of four star and receive little else. We would suggest that the clinics deliver more care than this and have the potential to provide a better service. They offer opportunities for social interaction in an unthreatening and familiar environment and the chance for friendship (we probably underestimate the important relationships patients form with one another and $63 \%$ of our patients met friends at the clinic). There is an attachment to a unit that will offer help, support and asylum throughout their lives and a sense of belonging for people who often belong nowhere. For some it may simply be a reason to go out of the house for the day. Attendance maintains the notion of accepting some responsibility for one's health, illness and treatment and provides access to medical and nursing staff who know their condition and are familiar with their individual case. We seriously doubt that treatment at home or health centre will provide this range of benefits and it may be our patients already know this. It is interesting, in this context, to note the findings of Holloway (1988) regarding the favourable opinions of new long-term patients attending day care who identified the important aspects of this facility to be staff support and the chance to meet people. He suggests that structuring of patients' time and shared experience outside the home were valuable and that day care for the chronically sick directed solely at treatment may be less successful in meeting the needs of the chronic patient. We support Dr Holloway's views in this respect, which are pertinent to the depot clinic and concur with the recommendation for further evaluation of patients' opinions of treatment facilities.

However, the clinic was obviously not everyone's cup of tea. We must acknowledge that a proportion of patients were unhappy with it to the extent of preferring an alternative arrangement and this emphasises the need to provide a diversity of treatment approaches within a flexible service. Offering the consumer choice of types of care is an important part of good community care and clearly patient satisfaction will influence treatment compliance. Indeed, flexibility already operates to some degree in that some patients will be receiving their treatment at home, from GP etc., depending on their need, and this is the way it should remain. For those patients who might prefer or benefit from the clinic arrangement this should remain available although a number of improvements need to be made. We strongly recommend attention to improved surroundings, decor and furnishings; adequate consultation room for private interview and acceptable social waiting areas; more staff, including use of volunteers and ensuring the patient's own CPN delivers treatment. A regular senior medical input should be present, as well as time from clinical psychology and a social worker accessible to discuss social problems or for family work with other members of the multidisciplinary team. This setting provides an ideal base for family support groups, family intervention and involvement with care and there is no reason why relatives could not attend in the same way they attend any other outpatient clinic. There is potential for development of the social milieu, provision of light refreshments and the possible addition of social events arranged for patients (with or without families) with a committee who would raise funds. Last, but certainly not least, a far greater attention should be paid to staff morale. Many of the problems of depot clinics simply reflect the larger issue of neglect of the care of the chronic patient and similar neglect of the staff who care for them. Continued deprecation of the services does not help and we were pleased to find that staff were interested to hear of these results, staff usually short of positive reinforcement.

Notwithstanding the methodological simplicity of this study, it would seem that the depot clinic has some supporters. We advocate the retention of these underrated facilities but as part of a flexible, comprehensive service and believe there is scope for considerable improvement and innovation in their delivery of care.

When proposing the replacement of a service more attention needs to be given to identifying therapeutic benefits offered by the existing service, not just its deficiences, and the consumers' opinion could be a valuable part of this appraisal. The chance of losing these benefits in the process of change must be carefully considered and balanced against the advantages offered by a new alternative. If real therapeutic benefits go unrecognised and are lost we may find that we have successfully thrown the baby out with the bathwater.

\section{References}

BEVERIDGE, A. (1987) Are depot clinics out of date? Bulletin of the Royal College of Psychiatrists, 11,92-93.

Holloway, F. (1988) Psychiatric day care: views expressed by chronically ill patients. Health Trends, 20, 34.

KEAN, P. \& FAHY, T. J. (1982) Who receives the aftercare? Utilization of services by discharged patients. Psychological Medicine, 12, 891-902.

MURPHY, E. (1988) Community care II: possible solutions. British Medical Journal, 296, 6-8. 